\title{
The Technological Conundrum: How Rapidly Advancing Technology Can Lead to Commoditization
}

\author{
Tat Y. Chan ${ }^{1} \cdot$ Ravi Dhar $^{2} \cdot$ William Putsis $^{3}$
}

Published online: 17 April 2015

(C) Springer Science+Business Media New York 2015

\begin{abstract}
Much of the recent empirical IO research has been conducted in the context of relatively mature, stable (often consumer packaged goods) markets. In these markets, consumer preferences and competitive interaction are often characterized by relatively stable patterns over time. In contrast, modeling and estimating analogous patterns in rapidly changing technology-intensive industries, where brand preferences and technology evolve rapidly, can be challenging. Our primary objective in this research is to build on recent developments in econometric estimation and modeling to assess the time-varying shifts in consumer brand preferences in a rapidly changing technology industry. In doing so, we document an interesting technological conundrum-technological advancement can often lead to commoditization. In the Personal Computer (PC) market, this began with differentiation that was initially central to success in a market that has been
\end{abstract}

We have been benefited from discussions with Ambar Rao, Chakravarthi Narasimhan, Seethu Seetharaman, Boudhayan Sen, and Vishal Singh. The usual disclaimer applies.

William Putsis

William_Putsis@unc.edu

Tat Y. Chan

Chan@olin.wustl.edu

Ravi Dhar

Ravi.Dhar@Yale.edu

1 Washington University in St Louis, Campus Box 1133, One Brookings Drive, St Louis, MO 63130-4899, USA

2 Yale School of Management, Yale University, 135 Prospect Street, New Haven, CT 06520-8200, USA

3 Kenan-Flagler Business School, University of North Carolina at Chapel Hill, CB 3490, McColl Building, Chapel Hill, NC 275993490, USA increasingly commoditized over time. Methodologically, we adopt a factor-analytic approach to model and estimate the evolution of consumer brand preferences over time.

Keywords High-technology markets · Brand strategy · Evolving consumer preferences $\cdot$ Industry life cycle

\section{Introduction}

Much of the recent empirical IO research has been conducted in the context of relatively mature, stable consumer packaged goods markets. In these markets, consumer preferences and competitive interaction are often characterized by relatively stable patterns over time. In contrast, modeling and estimating analogous patterns in rapidly changing technology-intensive industries (e.g., mobile phones, personal computers, etc.) can be challenging for a number of reasons. In these industries, we often observe the rapid introduction of new technologies early on in the industry's life cycle. As these new technologies are introduced and as these markets evolve over time, consumer brand preferences in the market are likely to be evolving over time as well. For example, in the early stages, consumers may be concerned with manufacturers' market presence (e.g., will they still be around in the future). As the industry develops over time, some technologies begin to emerge as the industry standard and consumer preferences typically migrate to those manufacturers whose products are compatible with emergent standards and hence would be easier to use (e.g., the Windows GUI, Intel Inside, etc.). This may lead to two consequences. First, nearly all brands adopt the same dominant technologies that consumers prefer, i.e., the industry became "standardized" in the technology level (e.g., "Wintel"). Second, since product innovation can no longer be sustainable as a source of differentiation across brands in the marketplace 
[20], consumers' perception of the differentiation of brand attributes (e.g., customer services and after-purchase technical support) and/or their preferences for those attributes may evolve, often quite dramatically, across the key stages of the industry life cycle. Consequently, due to the nature of these industries and how they evolve over time, the traditional assumption of time-invariant brand preferences is often not tenable. Thus, the interplay of demand-side influences and competitive interaction across firms occurs along multiple dimensions and evolves over time, giving rise to a number of important theoretical and methodological challenges.

Using this general description of industry development over time as motivation, our primary objective in this research is substantive in nature - to build on recent developments in econometric estimation and modeling to document the timevarying shifts in consumer brand preferences in a rapidly changing technology industry, using the PC industry as an example of how one can obtain substantive insights into the development of an industry over time.

Methodologically, we adopt a factor-analytic approach to model the evolution of consumer brand preferences over time. We develop two alternative models. The first model assumes that consumers' preference weights for brand attributes are evolving while brand attributes are constant. The second assumes that brand attribute values are time varying but not for consumers' preference weights. We focus on the personal computer industry in the USA as an empirical application, in large part due to the availability of data early on in the industry's lifecycle, although the methodology would be appropriate for understanding the evolution of consumer brand preferences over time for any part of the world. In our exploratory empirical analysis, we use our model to address two specific and important research questions: first, what drives the changes in consumer preferences for PC manufacturer brands over time? Second, how do these changes impact the cross-brand substitutability and hence the competition across manufacturers in the market?

We estimate the two competing models. We then test these two models using the non-nested Cox-type test prosed by Smith [18]. Test result supports the model allowing for the dynamic change in the preference weights for brand attributes. Estimation results from this model show that the positions of the major PC brands were located much closely in the 1990s than in the mid 1980s, implying an increase in cross-brand substitutability as perceived by consumers over this time period. This finding offers an explanation for the intense price competition between PC manufacturers that we observe starting in the early 1990s. It is also consistent with the more widespread belief that the PC industry has become "commoditized" since that period due to the dominance of the "Wintel" technologies. Further, we find that consumer preference weights evolved in a significantly different way across different brand-level attributes. This evolution in the industry structure and consumer preferences cannot be inferred from standard empirical models using a static framework. Thus, we believe that there are important substantive contributions to be derived from our research.

The contributions in this paper include the following:

1. The development of a methodology to infer the timevarying changes in consumer preferences over time from market-level aggregate data.

2. The study of the impact of the changes in consumer brand preferences on the degree of substitutability between different manufacturer brands over time.

3. Substantively, by identifying the above changes, one will gain better insights into the nature of competition in the rapidly evolving technology markets.

It is important to note that previous research has tried to incorporate dynamics in consumer choice models in various ways. For example, some researchers have done this by allowing for serial correlated error terms (e.g., [1]) or state dependence (e.g., [17]) directly in the utility function. Lachaab et al. [14] model consumer preferences using a Bayesian state-space approach, focusing on observed product attributes. In our model, since our objective is to substantively understand and document preferences and their interplay with competitive effects, we remain agnostic to the source of evolution and let the data inform our conclusions; hence, our emphasis is on the evolution of preferences on latent brand attributes with the objective of inferring how firm competition in the industry was driven by these changes. ${ }^{1}$

Our modeling approach is based on the factor-analytic model in Elrod [9] and Elrod and Keane [10], where their main objective is to infer consumer preferences for the latent brand attributes. ${ }^{2}$ They assume that each brand has a unique position in the multi-dimensional attribute space that is unobserved to researchers. Our model differs in that our main focus is the evolution of consumer brand preferences, which complicates the cross-brand substitution patterns. It is important to note, however, that this represents an extension of existing methodology, when applied to data for a rapidly evolving technology market, can provide substantive insights into the market's development over time; although our empirical application is the PC industry, we believe that the methodology

\footnotetext{
${ }^{1}$ There are a number of other studies that also employ the state-space approach. For example, Van Heerde et al. [20] used a dynamic linear model (see [6]) to investigate how consumer preferences are impacted by product innovations. They found, for example, that innovations make brands more similar and decrease differentiation for existing brands. These results support our findings at a number of levels (we will provide details later in the paper).

${ }^{2}$ Other empirical studies using the same approach include Carroll et al. [5], Chintagunta [7], and Erdem and Winer [12]. Based on panel data, Erdem [11] also models how state dependence components affect consumer preferences for the latent attributes.
} 
developed in the paper could be applied to many other technology-based product markets.

The remainder of the paper proceeds as follows. Section 2 discusses the industry setting and describes the data. The empirical model and estimation method are described in detail in Section 3, while the estimation results are discussed in Section 4 . Section 5 concludes the paper.

\section{The Personal Computer Industry}

A personal computer can be defined as a general-purpose, single-user machine that is microprocessor based and can be programmed in a high-level language. For our purposes, information was collected from International Data Corporation's (IDC) Processor Installation Census (PIC). IDC is the oldest among the various firms that tracks the American computer industry and is widely recognized as having a very accurate picture of the activity in this industry, one that starts very early on in the industry's lifecycle. ${ }^{3}$

Our study population includes all firms that sold desktop personal computer in the USA during the period 1983-1994. We focus on these early years for a number of reasons. First, these 12 years represented a dramatic evolution of the industry (one can think of the market over this time period as a rapidly evolving technology market). The formative years in an industry's lifecycle are often the focus of research in marketing, e.g., diffusion studies, time to takeoff, and then impact of disruptive technologies. We follow that tradition here. From a different perspective, during this period, the industry was characterized by rapid expansion in sales: sales grew from under 1 million units in 1983 to over 16 million units by 1994. In addition, the number of competing firms in the market increased from just over 100 in 1983 to well over 300 by 1994. This affords us an opportunity to examine an industry during a period of explosive growth, when change was particularly evident, in some ways a "strong" test of our approach.

In terms of competition among PC manufacturers, we observe significant changes during this period. Compaq and Gateway entered the market in 1984, Dell in 1985, and Packard Bell in 1986. They started small, gained market share gradually, and all became important players in the market in the 1990s (for example, both Compaq and Packard Bell overtook IBM in market share in 1994). At the same time, some established brands such as Tandy significantly lost sales and became an insignificant player in the industry (see Fig. 1).

\footnotetext{
${ }^{3}$ Detailed discussions on the IDC data can be found in Bayus and Putsis [2] and Putsis and Bayus [16] as well as on the IDC web site www.idc. com. Excellent historical reviews of the personal computer industry are given in Langlois [15] and Steffens [19]. Perhaps the most comprehensive reference site for a chronology of the industry can be found at www. islandnet.com/ KPOLSSON/comphist/.
}

The personal computer industry also experienced a rapid evolution in the microprocessor technology and operating system environments over this period. Every 4 to 5 years, a new generation of microprocessor was introduced into the market aimed at replacing older technologies. As a result, the productlife cycle of each generation of microprocessors had a span of about 8 to 10 years (see Fig. 2). Also note that in general, it took 4 to 5 years before the sales of a new technology pass that of the older technology. Thus, this early period gives us an opportunity to examine a market during a period of rapid technological change on the hardware side. Further, on the software side, the time period spanned the original announcement of the Microsoft Windows graphical user interface in 1983 through the launch of Microsoft Windows NT in 1993 and the beta version of Microsoft Windows 95 in 1994. Thus, from a variety of perspectives, the nature of the industry over this time period is consistent with our objective of studying the evolution of consumer preferences in a rapidly changing technology industry. ${ }^{4}$

In this study, we focus our analysis on the seven main manufacturer brands over this period-IBM, Compaq, Dell, Gateway, Packard Bell (PB), Hewlett Packard (HP), and Tandy, those with the largest accumulated market share in our dataand group all others as the "other brands". 5 Most brands in this group are relatively unknown to consumers and have very small market share. Some brands (e.g., Commodore) were well established in the early $80 \mathrm{~s}$, but their sales declined fast since the start of our data, while others (e.g., Acer, Toshiba etc.) became more significant players in the market later but their sales were negligible during our sample period.

The data demonstrate an interesting change in the pattern of pricing competition in the industry over time. Before the 1990s, the seven top manufacturers enjoyed a significant price premium over the other brands for both frontier and older technologies ${ }^{6}$; however, starting in the early 1990 s, they became more aggressive in terms of pricing competition (Fig. 3). In the period 1992-1994, the observed price premium of the

\footnotetext{
${ }^{4}$ By the mid to late 1990 s, the industry had matured significantly and was certainly a relatively stable market in the early part of the new millennium. Further, although we have data after 1994, IDC altered its data collection methodology in 1994 and there are important differences in reported sales and pricing pre and post 1994 . Hence, it is difficult, if not impossible, to directly combine the two data sets in model estimation. We feel strongly that we should err on the side of being conservative with the data, using only data for which we have time-related consistency in the way the data was collected and measured.

${ }^{5}$ Another major brand in our sample period is Apple. Consumers who switch from Wintel to Apple have to invest in manufacturer-specific technology adoption. Apple is very different from the other seven brands from a number of perspectives; hence, consumer's choice of switching in this context can be very different from what is implied in our empirical model below. As a result, Apple is included in the "outside" option in the demand model below.

${ }^{6}$ We will provide detailed definitions for frontier and older technologies later in the paper.
} 
Fig. 1 Market share of the seven leading manufacturer brands

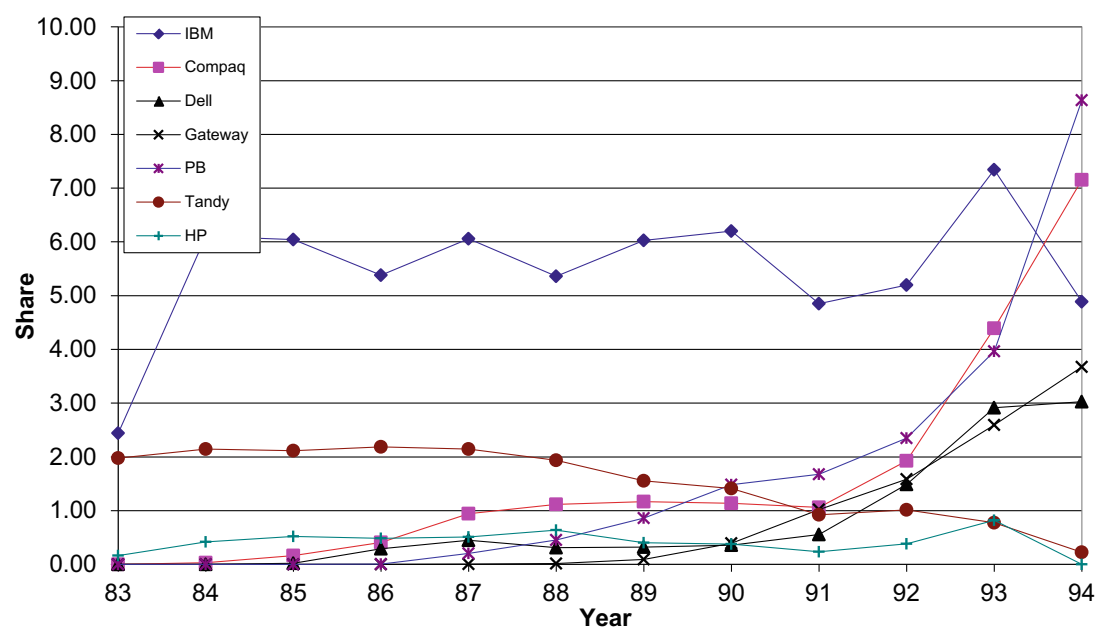

seven brands was virtually eliminated. In large part due to this strategic pricing change, brands such as PB, Compaq, Dell, and Gateway were growing at the expense of other less wellknown brand names. This evolution was dramatic, illustrating the rapid evolution in the PC market structure and why it is important to document and understand the basic driving forces behind the change in competitive strategies among PC manufacturers, the main focus of this research. We now turn to the model and empirical methodology to be employed.

\section{The Model and Empirical Methodology}

We model how evolving consumer brand preferences drive the change in the competitive environment in the PC industry from the 80 s to mid 90 s. We assume that when making purchasing decisions, consumers evaluate multiple latent attributes associated with each manufacturer's brand (e.g., perceived brand quality and services provided by manufacturers that are unobserved to researchers). Analogous to the factoranalytic model in Elrod [9] and Elrod and Keane [10], each brand has unique attribute values in the multi-dimensional attribute space, and consumers have preference weights for different brand attributes. The product of a brand's attribute levels and the corresponding preference weights for those brand attributes represent the consumer brand preference. Technology innovations (e.g., Intel's Pentium replacing the 486 technology; release of Windows 3.0 and Windows NT from Microsoft) and marketing efforts from upstream manufacturers (e.g. Intel's "Intel Inside" campaign) in the PC

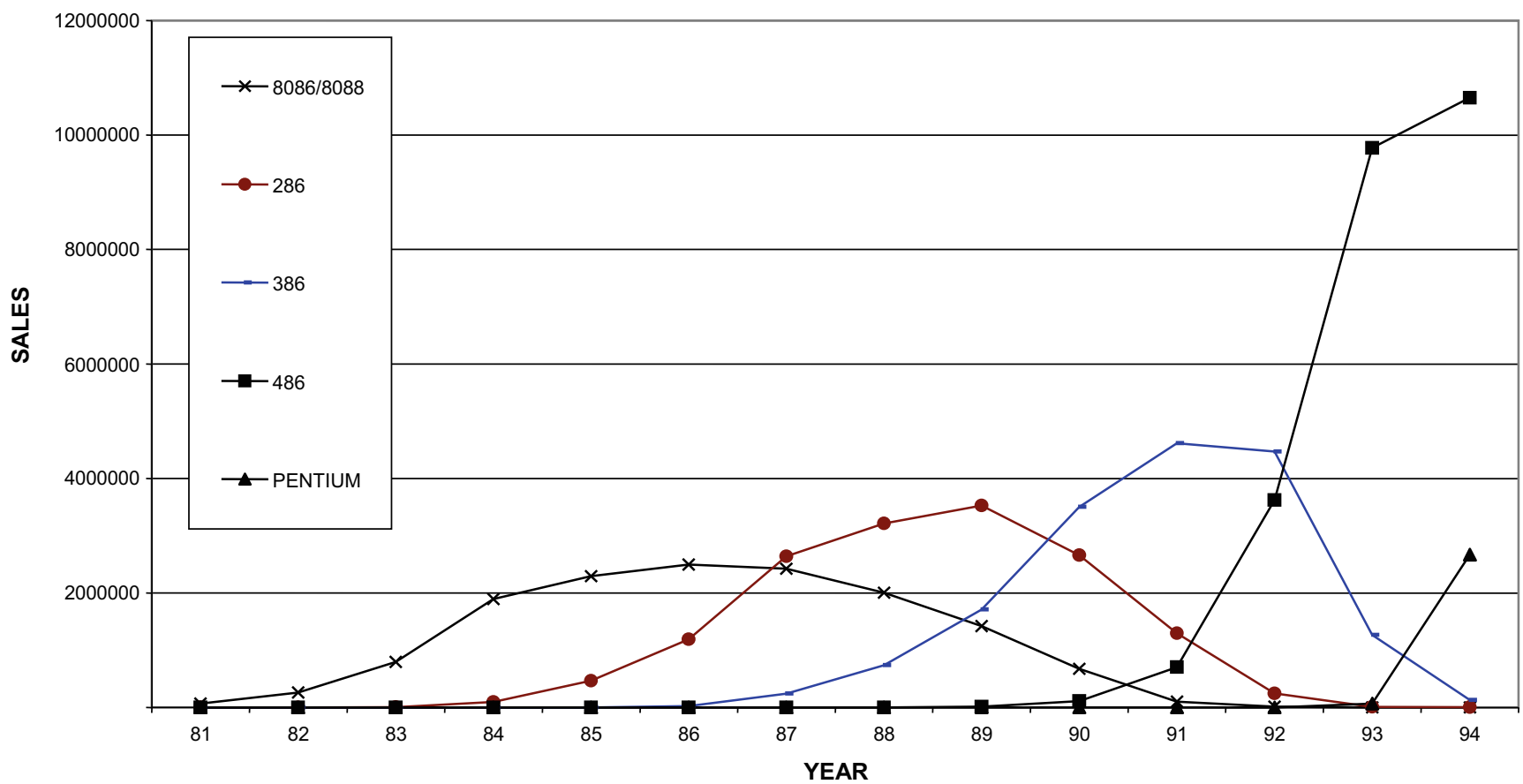

Fig. 2 PC sales of different microprocessor technologies 


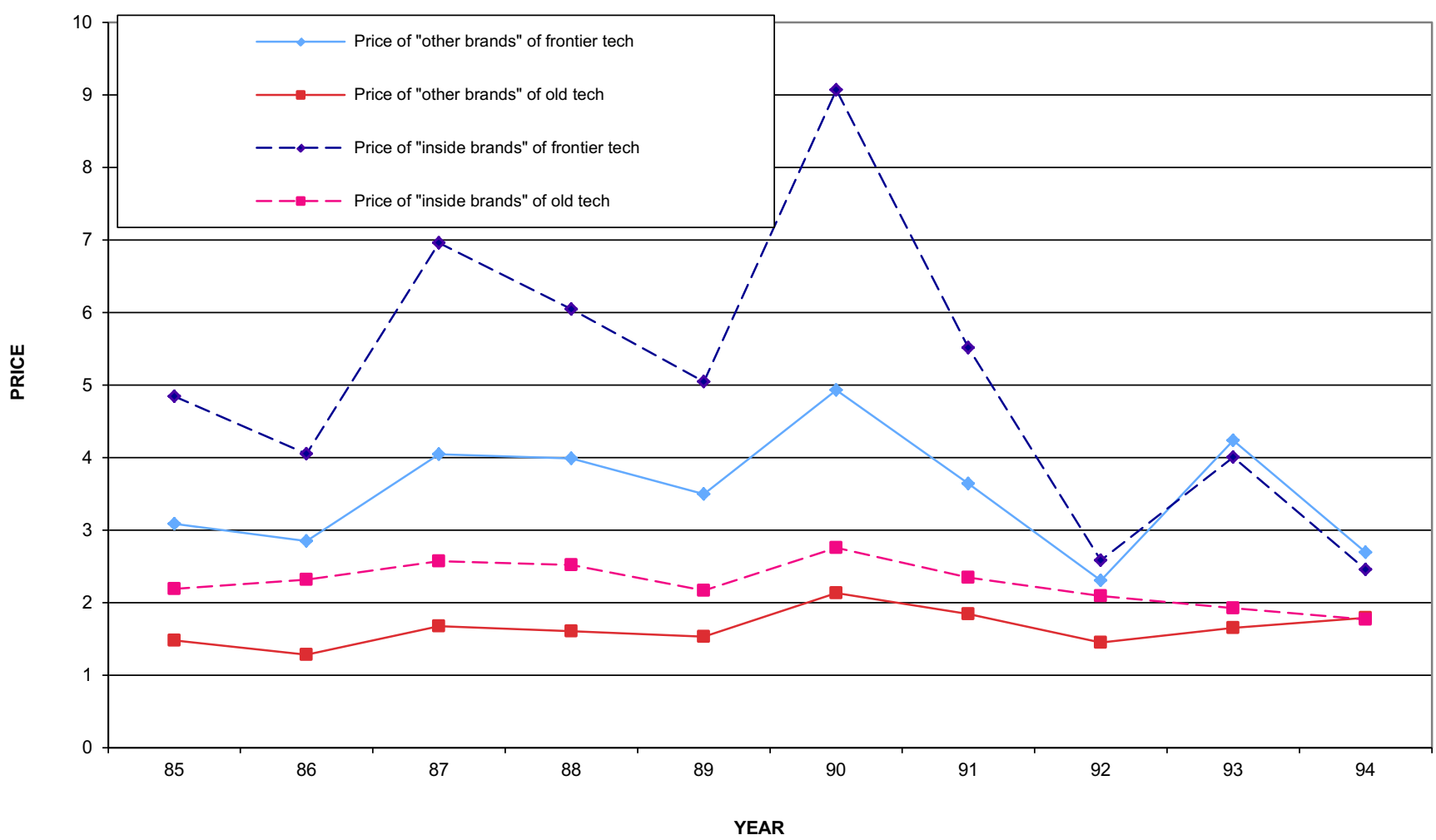

Fig. 3 Price convergence of seven national brands versus "other" brand prices

industry may have changed consumers' preference weights over time. For example, there may have been less concern about manufacturer brand quality as consumers came to realize that microprocessors and operating systems have the most important impact on machine performance; thus, consumers may have put more weight on service provisions from PC manufacturers. The advertising and product and service improvement efforts from a branded manufacturer, on the other hand, can change consumers' perception of the latent attributes of the brand over time.

\subsection{The Utility Model}

We allow for the fact that each manufacturer has a portfolio of differentiated products in the market in each period. In our data, there are altogether 6514 (product, year) combinations of observations. We begin by assuming that if consumer $i$ buys product $j$ at time $t$ with microprocessor technology $g$ from a manufacturer $m(m=1, \ldots, M$, including "other brands"), her indirect utility level will be equal to

$U_{i j t}=\lambda_{i t, m}+\kappa_{i t, g}+x_{j t} \beta-\alpha_{i} p_{j t}+\xi_{j t}+\varepsilon_{i j t}$

where $\lambda_{i t, m}$ denotes at time $t$ consumer $i$ 's preference for manufacturer brand $m$ and $\kappa_{i t, g}$ the preference for technology $g$ embedded in product $j$ at time $t$. Note that these preferences are allowed to be time varying and individual specific. The term $x_{j t}$ in Eq. (1) includes a vector of explanatory variables observed in the data. In our application, this includes the following variables: an intercept, year since $1983\left(Y R_{83}\right)$ and its square $\left(Y R_{83}{ }^{2}\right)$, quarter of the year when the model is introduced (DFI quarterly, which adjusts for seasonal effects), country of origin of the manufacturer $($ Foreign $=1)$, configuration $($ Tower $=1)$, unemployment rate, and GDP growth rate. We specify the price coefficient as $\alpha_{i}=\alpha_{0}+v_{i}^{\alpha}$, where $\alpha_{0}$ is the mean (negative) price coefficient to be estimated from the model and $v_{i}^{\alpha}$ a stochastic variable that we will discuss in more detail later. The term $p_{j t}$ is the (real) price of product $j$ at time $t$.

There are two components to the equation error structure. The first error term $\xi_{j t}$ captures consumer preferences for product features (such as memory and operating system) that we do not observe from data. This stochastic term will affect consumers' utility and also the pricing decisions of manufacturers. While the term $\lambda_{i t, m}$ in Eq. (1) captures brand-specific differences, $\xi_{j t}$ captures unobserved product differences not related to the individual brand. The second stochastic component, $\varepsilon_{i j t}$, is the idiosyncratic error assumed to be i.i.d. over $i, j$, and $t$. By standard assumption, the utility derived from an outside option is $U_{\text {iot }}=\varepsilon_{\text {iot }}{ }^{7}$.

Finally, we note that our model extracts away from the dynamics of consumer product replacement decisions of durable goods such as a personal computer (e.g., Gordon [13]). If consumers delay purchases in the expectation of technology

\footnotetext{
${ }^{7}$ The outside option is addressed in Footnote 5 above and will be further discussed later.
} 
innovations, our estimate of $\kappa_{i t, g}$ can be biased. Yet Fig. 2 shows that it takes 3 to 4 years for a new microprocessor technology to overtake older one in market share, suggesting that this may not be a major concern for the PC market during our sample period. Alternatively, if consumers delay purchases expecting price drops that are caused by technology innovations, the price coefficient $\alpha_{i}$ may be underestimated in our model. However, we believe that these factors should not have a major consequence for estimating the evolution of consumer brand preferences, which is the primary goal of this study. ${ }^{8}$

\subsection{Modeling the Evolution of Brand Preferences}

To model the time-varying and individual-specific brand preference $\lambda_{i t, m}$, the traditional approach is to assume a parametric distribution for the $M$-dimensional $\lambda$ 's overall brands, e.g., $\lambda_{t} \sim \operatorname{nnormal}\left(\overline{\lambda_{t}}, \Sigma_{\lambda}\right)$, focusing on how the mean brand preferences evolve over time. Estimating such a model is not an easy task because of an "over-parameterization" problem when the number of brands $M$ is large - consequently, researchers have had to rely on some parametric assumption on the evolution process of $\overline{\lambda_{t}}$ (for example, see Lachaab et al. [14] who used a state-space approach). In our application, we are employing market-level aggregate data, and consequently, we take a very different approach here.

Specifically, we assume in the model that there are $K$ brand-specific unobserved attributes. For example, if "quality" of brands and "services" provided by manufacturers are two perceived important attributes (which researchers do not observe from data) that consumers care about, then we would specify $K=2$ (in practice, $K$ is much smaller than the number of brands $M$ in the market). We model the consumer brand preferences through a factor-analytic approach similar to Elrod and Keane [10]; for manufacturer brand $m$, consumer $i$ 's preference is

$\lambda_{i t, m}=L_{m t} c_{i t}$

where $L_{m t}$ is a $1 \times K$ row vector of parameters, denoting the values that brand $m$ have for the unobserved brand attributes at time $t$ (and, thus, we will refer to $L$ as "the brand attribute level" throughout). The $K \times 1$ vector $c_{i t}$ represents the preference weights for these brand attributes that are specified as the following:

$c_{i t}=\bar{c}+\eta_{i t}$

\footnotetext{
${ }^{8}$ We also note that to estimate full heterogeneity and time evolution in consumer preferences for $x_{j t}$ (i.e., through $\beta$ ) is a non-trivial task. Since consumer preferences for the $x_{j t}$ themselves are not the focus of, nor generally material to the current study, we limit our approach in the interest of parsimony and in keeping the estimation manageable and focused at the issues at hand.
}

where $\eta_{i t}$ is assumed to be distributed as standard normal $N(0$, $I_{K}$ ), where $I_{K}$ is an identity matrix with dimension $K^{9}$ and $\overline{c_{t}}$ is a $K \times 1$ vector of parameters representing the average consumer preferences at time $t$. Therefore, we can re-write Eq. (2) as

$\lambda_{i t, m}=L_{m t} \overline{c_{t}}+L_{m t} \eta_{i t}$

To understand what drives the evolution of consumer brand preferences, it is substantively important to evaluate the changes in $L_{m t}$ and the changes in $\overline{c_{t}}$ over time. Yet Eq. (2') suggests that they cannot be separately identified in model estimation. This is because for any combination of $\overline{c_{t}}$ and $L_{m}$, another combination of estimates $\bar{c}$ will give the same result on the data generation process. ${ }^{10}$

We make use of a unique observation that can be used to test whether the changes in data are driven by either the timevarying preference weights for brand attributes or the timevarying brand attribute levels. Suppose it is the former reason. The relative position of brands in the position map will remain unchanged, since the change in $\bar{c}_{t}$ will impact the position of every brand in the same direction and magnitude, assuming that there are 3 years, two brands, and two latent brand attributes. In one scenario (scenario 1), preference weights for the brand attributes are $\bar{c}_{1 t}=\{1,3,2\}$ and $\bar{c}_{2 t}=\{3,1,4\}$ in the 3 years. Suppose brand attribute levels remain constant at $L_{1 t}$, ${ }_{1}=1$ and $L_{1 t, 2}=2$ for brand 1 , and $L_{2 t, 1}=2$ and $L_{2 t, 2}=1$ for brand 2. Since the relative magnitude of the attribute values of the two brands is constant, the left panel of Fig. 4 shows that the relative brand positions between the two brands will remain unchanged over time.

In another scenario (scenario 2), the preference weights for the brand attributes remain constant at $\bar{c}_{1 t}=1$ and $\bar{c}_{2 t}=2$. The brand attribute levels, however, are $L_{1 t, 1}=\{3,2,1\}$ and $L_{1 t}$, ${ }_{2}=\{1,2,3\}$ for brand 1 , and $L_{2 t, 1}=\{2,2,2\}$ and $L_{2 t, 2}=\{3,2,1\}$ for brand 2 , in the 3 years. The right panel of Fig. 4 shows that the positions of the two brands relative to each other will evolve. While the position of brand 1 on the dimension of attribute 1 declines over time, that of brand 2 remains constant and, while the position of brand 1 on the dimension of attribute 2 increases over time, that of brand 2 declines. This observation suggests that we can use a non-nested model test by first estimating a model of time-varying consumer preference weights for the brand attributes as the following:

Model 1: $\quad \lambda_{i t, m}=L_{m} \bar{c}_{t}+L_{m} \eta_{i t}$

\footnotetext{
${ }^{9}$ The standard normal distribution assumption follows Elrod and Keane [10], which is necessary for model identification.

${ }^{10}$ Suppose we have data on marketing efforts from upstream manufacturers (e.g. Intel's "Intel Inside" campaign) that should only impact the change in the preference weights for the brand attributes, $\bar{c}_{t}$, and data on marketing efforts from branded manufacturers, which should only have effect on the change in the brand attribute levels, $L_{m t}$. It is possible to identify both changes from model estimation. In this study, however, we do not have these data.
} 
Fig. 4 An illustration of changes in preference weights and brand positions

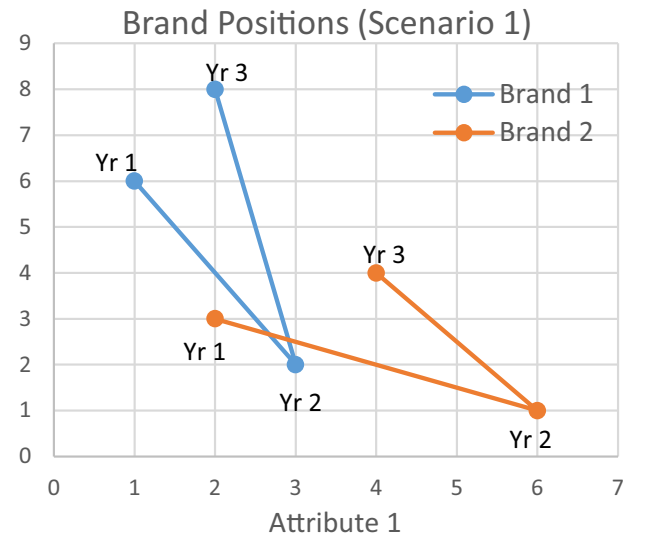

Here, we assume that brand attributes are fixed as $L_{m t}=$ $L_{m}$, and we estimate $L_{m}$ and $\bar{c}_{t}$ separately. We can then estimate the alternative model that allows for timevarying brand attributes as

Model 2 : $\quad \lambda_{i t, m}=L_{m t} \bar{c}+L_{m t} \eta_{i t}$

In this specification, $\bar{c}$ is estimated as a time-invariant parameter and $L_{m t}$ are estimated for each manufacturer brand in each time period. ${ }^{11}$ Since model 1 imposes more restriction on how brand positions evolve in the position map, model 2 will always perform better in terms of data fit. We can test whether or not model 1 can be rejected by comparing its performance (based on the criterion function value) with model 2 . If we cannot reject model 1, it implies that the evolution of brand preferences is likely to be driven by the changes in preference weights; otherwise, the evolution is at least also driven by the changes in brand attribute levels.

In practice, however, it is difficult to estimate $L_{m t}$ for all manufacturer brands and all time periods because of the large dimensionality of the parameter space. ${ }^{12}$ As we will discuss below, brand attribute parameters have to be estimated non-linearly, which is computationally very costly. Because of this reason, we estimate a restricted version of model 2 assuming that attribute $k$ of brand $m$ follows a linear dynamic change as

$L_{m t, \mathrm{k}}=L_{m, k}^{0}+L_{m, k}^{1} \times t_{83}$

where $L_{m, k}^{0}$ and $L_{m, k}^{1}$ are parameters to be estimated and $t_{83}$ represents the number of year after 1983 (the starting year of our sample) and, if a personal computer manufacturer operated after 1983, $t_{83}$ is the number of years of its existence.

\footnotetext{
$\overline{{ }^{11} \text { To estimate }}$ both model specifications, further normalization restrictions are required. Details are below.

${ }^{12} \bar{c}_{t}$ in Model 1 can be estimated for each attribute in each period, assuming that the number of attributes $K$ is much smaller than the number of manufacturer brands $M$ (7 in the study).
}

Such a specification fully captures how brand attribute levels dynamic changes under scenario 2 in Fig. 4; however, it cannot describe well the changes in consumer brand preferences under scenario 1 , because the preference weights $\bar{c}_{1 t}$ and $\bar{c}_{2 t}$ do not move linearly. Instead, model 1 is a better model specification for the scenario. As a result, we have two competing non-nested models. We estimate model 1 and the restricted model 2 separately. We then conducted a non-nested model test to decide which specification better fits with the data. If the effect of the changes in preference weights is dominant on the evolution of consumer brand preferences, test result will be in favor of model 1 . If the effect of the changes in brand attribute levels is dominant, model 2 will be chosen in the test. Details will be discussed in a later section.

To estimate the two models, we need to place further identification restrictions in Eqs. (4) and (5). In the simple case with $K=2$, we impose the following restrictions:

(a) Brand attributes levels $L_{m}$ for at least one brand are zero, that is, in the market position map, this brand is located at the origin $(0,0)$. In model estimation, we assume that all manufacturer brands other than the top seven brandsCompaq, Dell, Gateway, PB, Tandy, HP and IBM - are located at the origin ("other brands"). Such normalization implies that our estimated $L_{m t}$ for the top seven brands are relative to all other brands. ${ }^{13}$

(b) One of the attribute values for another "inside" brand is fixed to be equal to one. In model estimation, we choose the value of the second brand attribute of IBM, a longexisting major brand, for the normalization. That is, $L_{I B M}$, ${ }_{t, 2}=1$. This choice should not matter for the estimation results since consumer preferences $\bar{c}_{t}$ will be scaled to adjust for the normalization.

\footnotetext{
${ }^{13}$ The mean value of these normalized brands in consumer utility will be captured by the intercept $x_{j t}$ in equation (1). The heterogeneity in brand values among these brands will be absorbed by demand shocks $\xi_{j t}$.
} 


\subsection{Other Components in the Utility Function}

Consumers' purchase utility depends on not only the brand but also the performance and the price of a computer. For performance, we focus on the microprocessor technology, which is the most relevant attribute. We model how consumers' preferences for a microprocessor technology evolve over time. The most important microprocessor manufacturer is Intel, which produced chips that were used widely by all top PC manufacturers except (until recently) Apple. We assume that consumer's preference for technology $g$ embedded in product $j$ at time $t, \kappa_{i t, g}$, has two components: first, there is an inherent value associated with the technology, and we expect this value to be higher the later the technology was introduced. In our data, there are six major generations including (i) Z80, (ii) $8080 / 8085 / 8086 / 8088$, (iii) Intel 286, (iv) Intel 386, (v) Intel 486, and (vi) Intel Pentium.

We also include preferences for "sub-technologies"-DX and SX models - for Intel 386 and 486 in our model. Second, to capture the change in the value of using each technology, we assume that there is additional utility if $g$ belongs to the frontier technology, i.e., the leading technology in the market at time $t$. In our data, the frontier technology was 286 from 1983 to 1986, 386 from 1987 to 1989, 486 from 1990 to 1992, and Pentium from 1993 to $1994 .{ }^{14}$ We specify the technology preference function as the following:

$\kappa_{i t, g}=\rho_{g}+\tau_{i t} \cdot 1\left\{F_{g t}\right\}$

where $\rho_{g}$ represents the consumer preference for technology $g$, which for simplicity is assumed to be neither individual specific nor time varying. The variable $1\left\{F_{g t}\right\}$ is an indicator equal to 1 if $g$ is "frontier" in period $t$ and 0 otherwise. We specify $\tau_{i t}$ as an exponential function of the duration since $g$ has been introduced (to capture the "newness" of the frontier technology), $t_{g, I N T R O}$, and the number of years since our sample period starts in 1983 (to capture the change in consumers' preference for new technology over years), $(t-83)$. That is,

$\tau_{i t}=\exp \left[\tau_{0}+\tau_{1} \cdot t_{g, I N T R O}+\tau_{2} \cdot(t-83)+v_{i}^{\tau}\right]$

where $v_{i}^{\tau}$ is an individual-specific random variable. ${ }^{15}$

The price coefficient $\alpha_{i}=\alpha_{0}+v_{i}^{\alpha}$ in Eq. (1) represents the disutility of the cost of purchase. We allow that the preference for performance and the disutility for cost to be correlated among individual consumers. Conceptually, consumers who prefer

\footnotetext{
14386 and 486 were introduced into the market late in the years 1986 and 1989 , respectively. There were very few PC products incorporating these two technologies in their introduction years. Hence, 286 and 386 were still the frontier technology in years 1986 and 1989, respectively.

${ }^{15}$ Although $\tau_{i t}$ is interpreted as the preference for frontier technology, it can also be interpreted as the value loss of a technology once it is replaced by a faster and more powerful microprocessor technology, e.g., some software are designed for the new one but are incompatible with old ones.
}

frontier technology (innovators) may have different price sensitivity from those who are more risk averse to new technology. It is also likely that in our data, business buyers and individual home users may be different in technology preference and price sensitivity. To model the correlation, we assume that $v_{i}^{\alpha}$ and $v_{i}^{\tau}$ are jointly distributed as

$\left(v_{i}^{\alpha}, v_{i}^{\tau}\right)^{\prime} \sim N\left(0, \Sigma_{\alpha, \tau}\right)$

and estimate the variance-covariance matrix $\sum_{\alpha, \tau}$ including parameters var(price), cov(price, frontier), and var(frontier). Assuming $\alpha_{0}$ is positive (as there is a negative sign on $\alpha_{i}$ in Eq. (1)), the lower the value of $v_{i}^{\alpha}$, the less price sensitive is consumer $i$. If $\operatorname{cov}$ (price, frontier) is negative, the consumer places a higher value for the frontier technology. This is consistent with conventional belief that first adopters of new technology are less sensitive to price.

\subsection{Model Estimation}

Assuming a Gumbel distribution for $\varepsilon_{i j t}$ in (1), we have a multinomial Logit demand model. Let $s_{j t}$ be the market share of product $j$ at $t$. Suppose the product is embedded with technology $g$ and produced by manufacturer $m, m=1, \ldots, M$, including "other brands". Substitute Eqs. (2) to (6) into Eq. (1) and let ${ }^{16}$

$$
\begin{aligned}
\delta_{j t, m g}= & x_{j t} \beta-\alpha_{0} p_{j t}+\rho_{g} \\
& +\left[\tau_{0}+\tau_{1} \cdot t_{g, I N T R O}+\tau_{2} \cdot(t-83)\right] \cdot 1\left\{F_{g t}\right\} \\
& +L_{m t} \bar{c}_{t}+\xi_{j t}
\end{aligned}
$$

be the "mean utility" among consumers, where the subscript " $m g$ " denotes that product $j$ belongs to manufacturer $m$ with technology g. Also let

$\omega_{i j t, m g}=L_{m t} \eta_{i t}-v_{i}^{\alpha} p_{j t}+v_{i}^{\tau} 1\left\{F_{g t}\right\}$

be the individual-specific stochastic part in the utility function. We can write down the expected market share function as the following:

$$
\begin{aligned}
E\left(s_{j t}\right)= & \int \frac{\exp \left(\delta_{j t, m g}\right) \cdot \exp \left(\omega_{i j t, m g}\left(\eta_{i t}, v_{i}^{\alpha}, v_{i}^{\tau}\right)\right)}{1+\sum_{g^{\prime} \in j^{\prime}, j^{\prime} \in l, l, l, 1, \ldots, M} \exp \left(\delta_{j^{\prime} t, l g^{\prime}}\right) \cdot \exp \left(\omega_{i j^{\prime}, t, g^{\prime}}\left(\eta_{i t}, v_{i}^{\alpha}\right)\right)} \\
& d \Phi_{\eta}\left(\eta_{i t}\right) d \Phi_{\alpha \tau}\left(v_{i}^{\alpha}, v_{i}^{\tau}\right)
\end{aligned}
$$

wherein the denominator $g^{\prime} \in j^{\prime}$ denotes that technology $g^{\prime}$ is embedded in product $j^{\prime}$ and $j^{\prime} \in l$ denotes that product $j^{\prime}$ is produced by manufacturer $l$. In the denominator, " 1 " denotes the value of the "outside" option, as noted above. Finally, $\Phi_{\eta}$ is the (standard normal) distribution function of the $K$-dimensional $\eta_{i t}$ and $\Phi_{\alpha \tau}$ is the joint (normal) distribution function of $v_{i}^{\alpha}$ and $v_{i}^{\tau}$.

\footnotetext{
${ }^{16}$ For the simplicity of illustration, we still use $L_{m t}$ to represent $L_{m}$ in model 1 , and $\bar{c}_{t}$ to represent $\bar{c}$ for model 2 .
} 
We use a nested procedure for model estimation similar to Berry et al. [3]. To estimate the model, we need to address the price endogeneity issue here since the unobserved product features $\xi_{j t}$ may very well be correlated with prices. ${ }^{17} \mathrm{We}$ choose different sets of variables for model estimation. Detailed discussion of the estimation procedure and the instruments used in the estimation is in the Technical Appendix.

\section{Results}

\subsection{A Non-nested Model Test}

We use GMM to estimate competing models 1 and $2 .{ }^{18}$ The criterion function value of model 1 is about $7 \%$ lower than that of model 2, showing a better performance based on moment conditions. To test two competing models $h$ and $h$ ', Smith [18] proposed a Cox-type non-nested model test when both models are estimated by GMM. Define $g_{h}$ and $g_{h}$, as the moment conditions and $V_{h}$ and $V_{h}$, the consistent estimators of the variance of the moments, models $h$ and $h$, respectively. Conditional on model $h$, the Cox-type statistic for model $h$ ' against model $h$ is the following:

$\mathcal{C}\left(h^{\prime} \mid h\right)=\frac{1}{N} g_{h}^{\prime} V_{h}^{-1} A_{h} g_{h^{\prime}}$

For our regression models, $A_{h}=\frac{1}{N} Z_{h}^{\prime} Z_{h^{\prime}} V_{h^{\prime}}^{-1}$, where $N$ is the number of observations and $Z_{h}$ and $Z_{h^{\prime}}$ are the sets of instrumental variables used for estimating models $h$ and $h$, respectively. Define $G_{h}=\nabla_{\theta_{h}}{ }^{\prime} g_{h}\left(\widehat{\theta}_{h}\right)$ as the derivative of $g_{h}$, evaluated at the estimates $\widehat{\theta}_{h}$, with respect to the parameter set $\theta_{h}$, and $M_{h}=I_{K_{h}}-G_{h}\left(G_{h}{ }^{\prime} V_{h}^{-1} G_{h}\right)^{-1} G_{h}{ }^{\prime} V_{h}^{-1}$, where $K_{h}$ is the dimension of $\theta_{h}$. Similarly, define $G_{h^{\prime}}$ and $M_{h^{\prime}}$ for model $h$ '. Theorem 2.1 in Smith [18] shows that

$\mathcal{C}\left(h^{\prime} \mid h\right) \rightarrow{ }_{L} N\left(0, \omega_{h}^{2}\right)$

where $\omega_{h}^{2}=g_{h}{ }^{\prime} V_{h}^{-1} A_{h} M_{h^{\prime}} V_{h^{\prime}} M_{h^{\prime}} A_{h^{\prime}} V_{h}^{-1} g_{h}$, if $\omega_{h}^{2}>0$.

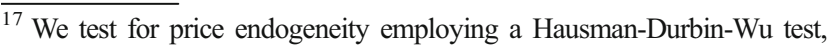
assuming that we have valid instruments for prices (see discussion below). The null hypothesis that price is exogenous can be rejected at $p=.05$.

${ }^{18}$ To calculate market share from sales, we need to know the potential market size for personal computers, which is not observed from the data. We do not want to impose the standard assumption in the literature that it is equal to the total population or the number of households, since consumers in our data also include firms, whose needs of PCs may vary significantly depending on their industries and business scale. Therefore, we assume that the potential market size in every year $t$ is equal to the total PC sales in 1999, 4 years after our sample period ends. We test the impact of such assumption on estimation results by re-estimating our model assuming the potential market size is $50 \%$ smaller and larger than this size. Our major results (e.g., time-varying brand preferences, price, and technology coefficients) remain qualitatively the same. Hence, we conclude that our key findings are robust to the potential market size assumption.
}

Based on this procedure, we use model 2 as the null. The Cox-type statistic is $\mathcal{C}($ Model $1 \mid$ Model 2$)=7.5 e^{-008}$, with standard deviation $\omega_{\text {Model } 2}=3.7 e-008$. Therefore, we can reject model 2 at a 0.05 significance level. Based on this result, we choose model 1 as our proposed model. That is, we assume that consumers' preference weights change over time, but the values of attributes of each brand remain constant. Estimation results discussed below therefore are based on model 1 .

\subsection{Basic Results}

Table 1 presents the results for the proposed model. In terms of what we will refer to as the "basic" variables, there is face validity in the results overall, which is important as these basic variables need to be consistent with expectations if the timevarying and more complicated aspects of the model are to be credible. For significant estimates, the variable Year- 83 is negative. This implies that, ceteris paribus, the importance of personal computer hardware (in relation to innovations in microprocessor technology and other product attributes) in the overall utility of consumers has declined over time. This is not surprising since personal computers have gone from a new and innovative technology to nearly commodity products over time. The estimated coefficient for $D F I$ Quarterly is negative reflecting the fact in data that if a model was introduced late in the year, the overall market share is lower for that model. The coefficient for the variable Origin is positive suggesting that personal computers manufactured abroad result in a higher mean utility over the sample period. Finally, the estimated coefficient for Unemployment Rate is negative and the coefficient for GDP Growth is positive (though statistically insignificant in our proposed model). Given that unemployment and economic growth are highly correlated, having the expected sign on both variables, but only one being statistically significant is again not surprising.

There are a number of key variables that were both central to the model development and to prior research. These too exhibit substantial face validity. We will discuss them one by one:

1. The later the year of introduction of a technology (from the earliest Z80 to the latest Pentium), the higher the overall preference, suggesting that models introduced in later years had a higher level of performance. Further, SX subtechnologies in 386 and 486 significantly reduced consumer preference, while 486 DX2 and DX4 significantly increased consumer preference. These are consistent with industry knowledge.

2. The estimated coefficient for the Frontier variable ( $\tau_{0}$ in Eq. (8)) is statistically significant and negative. The interpretation is that at the start of the sample period, the predicted mean consumer preference for a newly introduced 
Table 1 Model results, proposed model

\begin{tabular}{|c|c|c|}
\hline \multirow[b]{2}{*}{ Mean utility estimates } & \multicolumn{2}{|c|}{ Proposed model } \\
\hline & Estimates & Std. error \\
\hline Constant & 9.10 & 0.82 \\
\hline Year-1983 & -1.46 & 0.13 \\
\hline$(\text { Year-1983 })^{\wedge} 2$ & 0.01 & 0.01 \\
\hline DFI quarterly & -0.57 & 0.05 \\
\hline Origin & 1.17 & 0.12 \\
\hline Configuration & 0.08 & 0.15 \\
\hline Unemployment rate & -0.43 & 0.07 \\
\hline GDP growth & 0.01 & 0.05 \\
\hline $\mathrm{Z} 80$ & -2.08 & 0.40 \\
\hline $8086 / 8088$ & -3.05 & 0.32 \\
\hline 286 & -0.47 & 0.32 \\
\hline 386 & 3.76 & 0.35 \\
\hline 486 & 5.62 & 0.42 \\
\hline Pentium & 8.70 & 0.67 \\
\hline $385 \mathrm{SX}$ & -1.48 & 0.18 \\
\hline $486 \mathrm{DX} 2$ and DX4 & 1.55 & 0.23 \\
\hline $486 \mathrm{SX}$ & -1.04 & 0.23 \\
\hline Frontier & -0.93 & 0.37 \\
\hline Frontier $*$ (time since introduced) & -0.05 & 0.07 \\
\hline Frontier * (year-1983) & 0.15 & 0.05 \\
\hline Real price (negative) & 5.38 & 0.06 \\
\hline \multicolumn{3}{|l|}{ Random coefficient estimates } \\
\hline S.D. (price) & 3.22 & 34.84 \\
\hline Cov(price, frontier) & -0.34 & 0.01 \\
\hline S.D. (frontier) & 0.51 & 0.01 \\
\hline
\end{tabular}

technology was positive and equal to $e^{-0.05}=0.39$. However, consumer preference for any particular frontier technology decreased over time as we move away from the introduction date (the coefficients for Frontier* (time since introduced) are negative). This suggests that as software manufacturers were faster in complementing the frontier microprocessor technology, the practical usefulness of newly introduced technologies increased over time.

3. The estimated coefficient for Price is positive and statistically significant. Since there is a negative sign in front of the coefficient (Eq. (1)), this implies downward-sloping demand curve, as expected. The consumer heterogeneity in price sensitivity (the variable S.D. (price)), however, is large. A significantly negative correlation between price sensitivity and frontier preference (the variable cov(price, frontier)) implies that consumers who prefer frontier technology are also less price sensitive, which is consistent with the conventional wisdom that innovators (as a customer type) prefer higher quality to low price. The result implies that for manufacturers, a price discrimination strategy is possible: they will have significant market power to charge higher price to innovator consumers if they can introduce new models incorporating the frontier technology earlier than competitors.

\subsection{The Evolution of Consumer Preferences}

Once we allow for time-varying preferences in the proposed model, we are able to document the changes that have occurred in consumer preferences for brand attributes over time, the primary objective of this research. Table 2 presents the estimated brand attribute values and changes in consumer preferences over time. Figure 5 presents the graphical brand attribute values that are represented by the two-dimensional "market map" representing the two latent attributes, "attribute 1 " and "attribute 2," both obtained from Table 2. Overall, the dispersion of brand attribute values in the map suggests a fair amount of brand differentiation and the existence of weaker and stronger brands. For example, Tandy is weak on both dimensions.

In principal, in analysis of this type, where two (or more) latent attributes are modeled and estimated, identifying these two, by definition unobserved, attributes can prove tricky. Although not central to the main objective of the paper, here, we use industry knowledge and discussions with industry experts to speculate that "attribute 1 " is closely aligned with "ease of use and compatibility" over this period, while "attribute 2 " is closely aligned with "service, support, and the ability to deliver." While speculative, these certainly make sense in light of changes in the industry over this time period. For example, Compaq and IBM are the strongest among the seven brands in terms of "ease of use and compatibility," but weak in "service, support, and the ability to deliver," while HP and Dell are the strongest in "service, support, and the ability to deliver".

Overall consumer preferences for brands depend on not only the brand attributes levels but also preference weights for the two attributes. The lower panel of Table 2 reports the estimated preference weights in every year from 1983 to 1994. Figure 6 presents a graphical representation of the shifts over time for these two attributes. This figure clearly demonstrates an interesting pattern over time: the estimated consumer preference for attribute 1 ("ease of use/compatibility") steadily increased until 1987 and then fell; in stark contrast, consumer preference for attribute 2 ("service/support/ability to deliver") first declined then rose starting in 1987. At the outset of the PC industry, service, support, and the ability to deliver were important considerations among consumers (e.g., "no one ever got fired for buying an IBM") - note the relative importance of this in 1983 in Fig. 6. As the industry developed, the importance of the microprocessor technology, operating system, and application software became increasingly important, 1987 coincided with the growth of the Windows operating system, 
Table 2 Brand attributes values and evolution of consumer preferences (proposed model only)

\begin{tabular}{|c|c|c|}
\hline Brand values for attribute 1 : & Estimates & Std. error \\
\hline Compaq & 8.08 & 0.42 \\
\hline Dell & 5.91 & 0.82 \\
\hline Gateway & 2.97 & 0.48 \\
\hline PB & 3.41 & 0.33 \\
\hline Tandy & 4.97 & 0.35 \\
\hline HP & 7.67 & 0.35 \\
\hline IBM & 8.17 & 0.32 \\
\hline \multicolumn{3}{|l|}{ Brand values for attribute 2 : } \\
\hline Compaq & 0.17 & 0.48 \\
\hline Dell & 1.91 & 0.49 \\
\hline Gateway & 1.00 & 0.54 \\
\hline PB & 1.04 & 0.47 \\
\hline Tandy & 0.52 & 0.31 \\
\hline HP & 2.13 & 0.30 \\
\hline IBM & 1.00 & \\
\hline \multicolumn{3}{|c|}{ Time-varying preferences for attribute 1 : } \\
\hline 1983 & -0.783 & 0.038 \\
\hline 1984 & -0.356 & 0.027 \\
\hline 1985 & 0.049 & 0.023 \\
\hline 1986 & 0.115 & 0.020 \\
\hline 1987 & 0.651 & 0.018 \\
\hline 1988 & 0.523 & 0.016 \\
\hline 1989 & 0.250 & 0.016 \\
\hline 1990 & 0.151 & 0.015 \\
\hline 1991 & 0.024 & 0.014 \\
\hline 1992 & -0.152 & 0.013 \\
\hline 1993 & -0.037 & 0.012 \\
\hline 1994 & -0.464 & 0.011 \\
\hline \multicolumn{3}{|c|}{ Time-varying preferences for attribute 2 : } \\
\hline 1983 & 3.278 & 0.178 \\
\hline 1984 & 1.734 & 0.131 \\
\hline 1985 & 0.685 & 0.117 \\
\hline 1986 & -1.077 & 0.102 \\
\hline 1987 & -2.559 & 0.088 \\
\hline 1988 & -1.590 & 0.081 \\
\hline 1989 & -0.775 & 0.078 \\
\hline 1990 & -0.243 & 0.075 \\
\hline 1991 & 0.072 & 0.072 \\
\hline 1992 & 0.189 & 0.065 \\
\hline 1993 & -0.428 & 0.063 \\
\hline 1994 & 1.925 & 0.063 \\
\hline
\end{tabular}

making the PC accessible to the masses (the Windows GUI was first announced in 1983 and first shipped in 1985). It was also the year that the Intel 386 microprocessor was introduced. Consequently, attribute 1 ("ease of use and compatibility") became increasingly important with the growth of the Wintel technologies as concerns about hardware compatibility with industry standards were played out - note the overwhelming importance of this attribute in the mid to late $1980 \mathrm{~s} .{ }^{19}$ As the industry matured and moved into the early 1990s, PC software became more user friendly as the graphical GUI became commonplace and Intel microprocessors have become the standard in every PC brand. As software compatibility and standardization became commonplace, the capability of introducing new models incorporating the most advanced Wintel technologies could no longer serve to differentiate PC manufacturers. Service, support, and the ability to deliver (e.g., the growth of the Dell model; attribute 2) once again became the focal point for competition. These results, when taken from the "big picture" view, suggests that, in addition to the microprocessor technology development, changes in consumer preferences over time was another important factor underlying the change in the competition pattern in the PC industry.

Building on this, we examined the changes in, and dispersion of, brand positions or market structure early versus later in the sample (see Fig. 7). This is accomplished by multiplying the brand attribute values with consumer preference weights in 1987 vs. 1993. We find this figure startling - the level of dispersion in brand positions and in the perceived differentiation were relatively large in 1987. However, in 1993, all seven brands are crowded in a small space close to the origin (the position of "other brands"). Bresnahan and Greenstein [4] discussed the "competitive crashing" in the computer market in the early 1990s that firms previously targeting different segments now competed for the same customers; hence, more aggressive competition was observed among PC manufacturers and the channel power has shifted toward the "Wintel platform." Such discussion is analogous to our finding in Fig. 6. Movement toward less differentiation, more tightly compacted brand positions in the industry structure is a consistent finding throughout all the results that allow for time-varying preferences over time and is one of the key insights gained out of an analysis of this type.

\subsection{Changes in Price Elasticities over Time}

To investigate the impact of the change in consumer preferences on the substitutability across brands, we calculated the relevant own- and cross-price elasticities over time. We first estimated the own- and cross-price elasticities among the seven brands and then between these and the "other brands" in the market. From years 1983 to 1994, we increased and decreased the observed prices of all models of each brand by $1 \%$ and then use the estimated changes in market share of all brands to compute the price elasticities. Finally, we computed

\footnotetext{
${ }^{19}$ Note that IBM and Compaq are leaders in attribute 1. IBM was the first PC manufacturer using operating systems from Microsoft. Compaq was one of the first who pushed out new PC models incorporating Intel 386 microprocessor in 1987.
} 


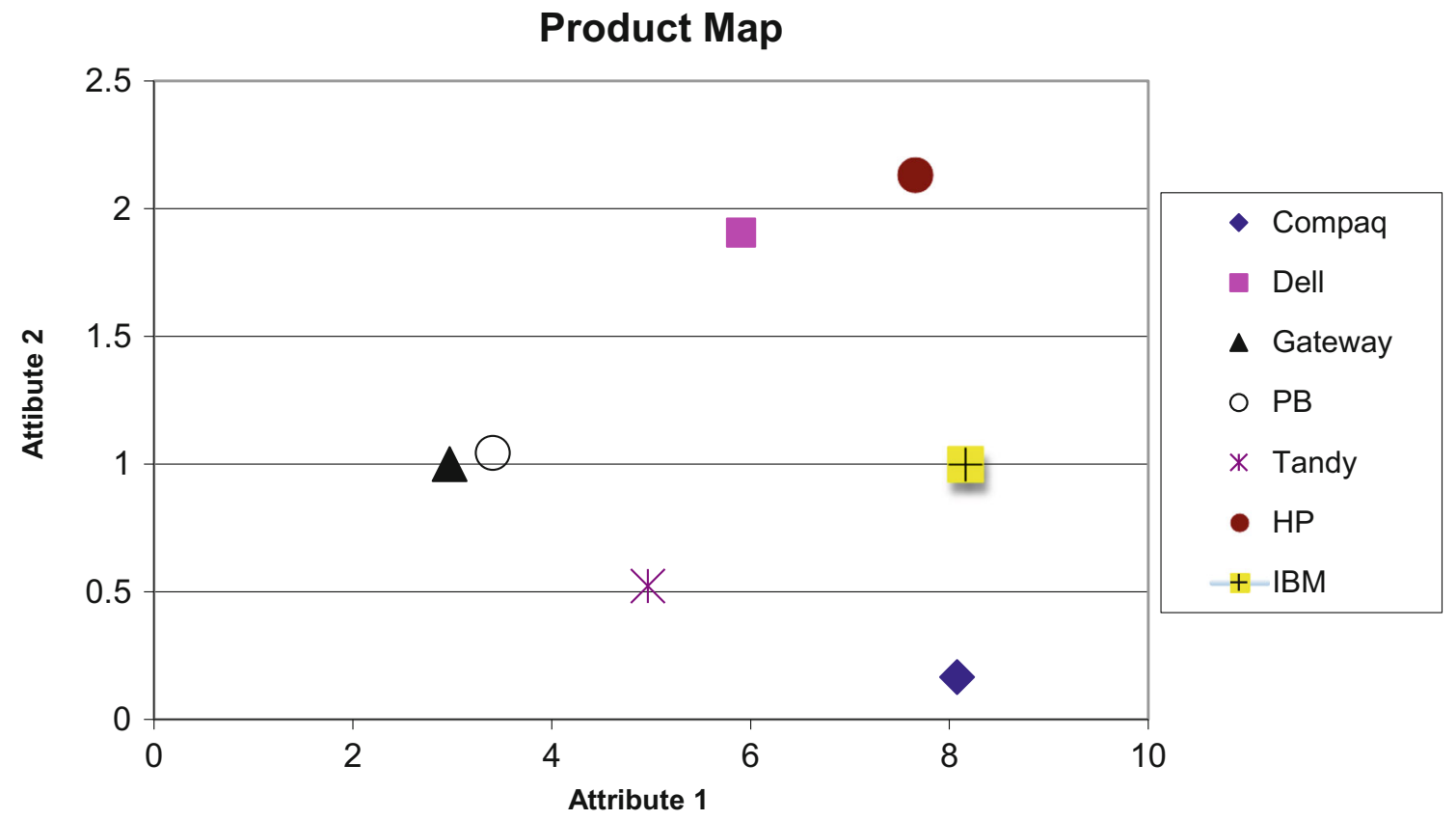

Fig. 5 Market positions of the seven manufacturer brands. Attribute 1 ("ease of use/compatibility"), attribute 2 ("service/support/ability to deliver")

an industry aggregate measure by calculating the average own- and cross-price elasticities weighted by the market share of each brand in each year based on the above-estimated elasticities for individual brands. The results are reported in columns $2-4$ in Table 3 using our proposed model.

Several interesting results are observed from the table. Perhaps most notable is the fact that the own-price elasticities in 1991-1994 are much higher than the entire 1980s (column 2). Furthermore, the estimated cross-elasticities with other included brands (column 3) as well as with "other brands" (column 4) are also higher in the later years of the sample. This is consistent with the earlier results that overall brand differentiation became smaller over time (see Fig. 7). This is also consistent with the industry belief that personal computer manufacturer brands were "commoditized" starting from early 1990s (possibly due to the "Intel Inside" and other marketing campaigns from upstream manufacturers such as Intel and Microsoft), thereby intensifying the competition between manufacturer brands (see Putsis and Dhar 1999). This lack of brand differentiation does not coincidentally coincide with the convergence of prices across manufacturers over the 1992-1994 period (see Fig. 4 above); taken together, this provides strong evidence that the change in pricing competition pattern in the 1990s has been driven by the increasing substitutability between brands as perceived by consumers.

Although the time-varying results demonstrate significant and clear changes in the underlying industry structure, we want to ensure that they are not due to other forces such as technology innovation (as new microprocessor technologies were introduced in different periods) or changes in product line portfolios (as new models enter and old models exit in every year). Therefore, we also addressed the following
Fig. 6 Estimated consumer preference weights. Attribute 1 ("ease of use/compatibility"), attribute 2 ("service/support/ ability to deliver")

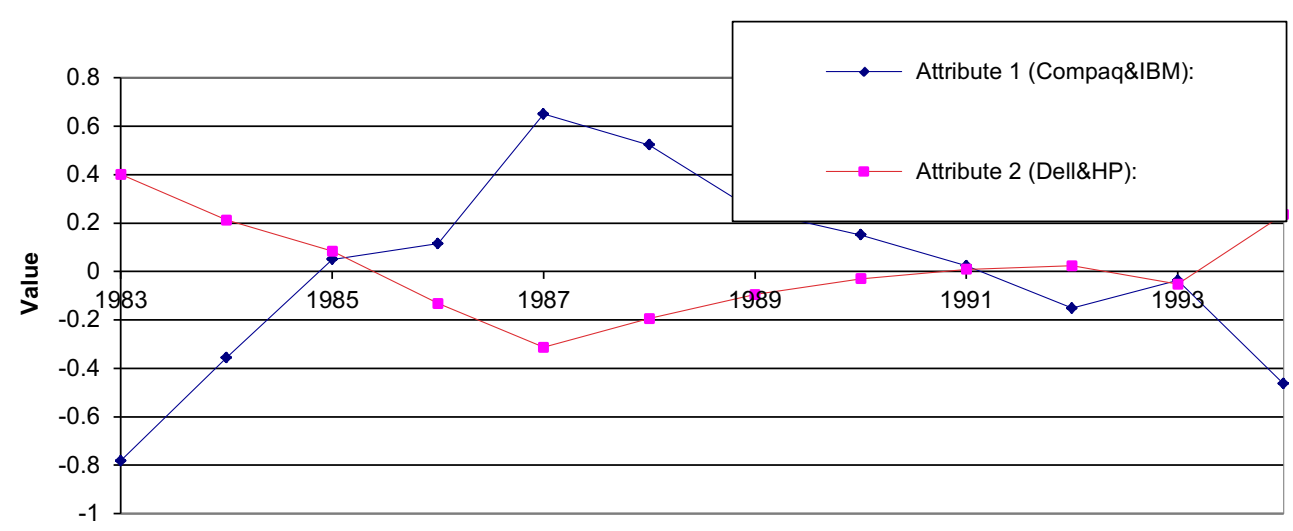




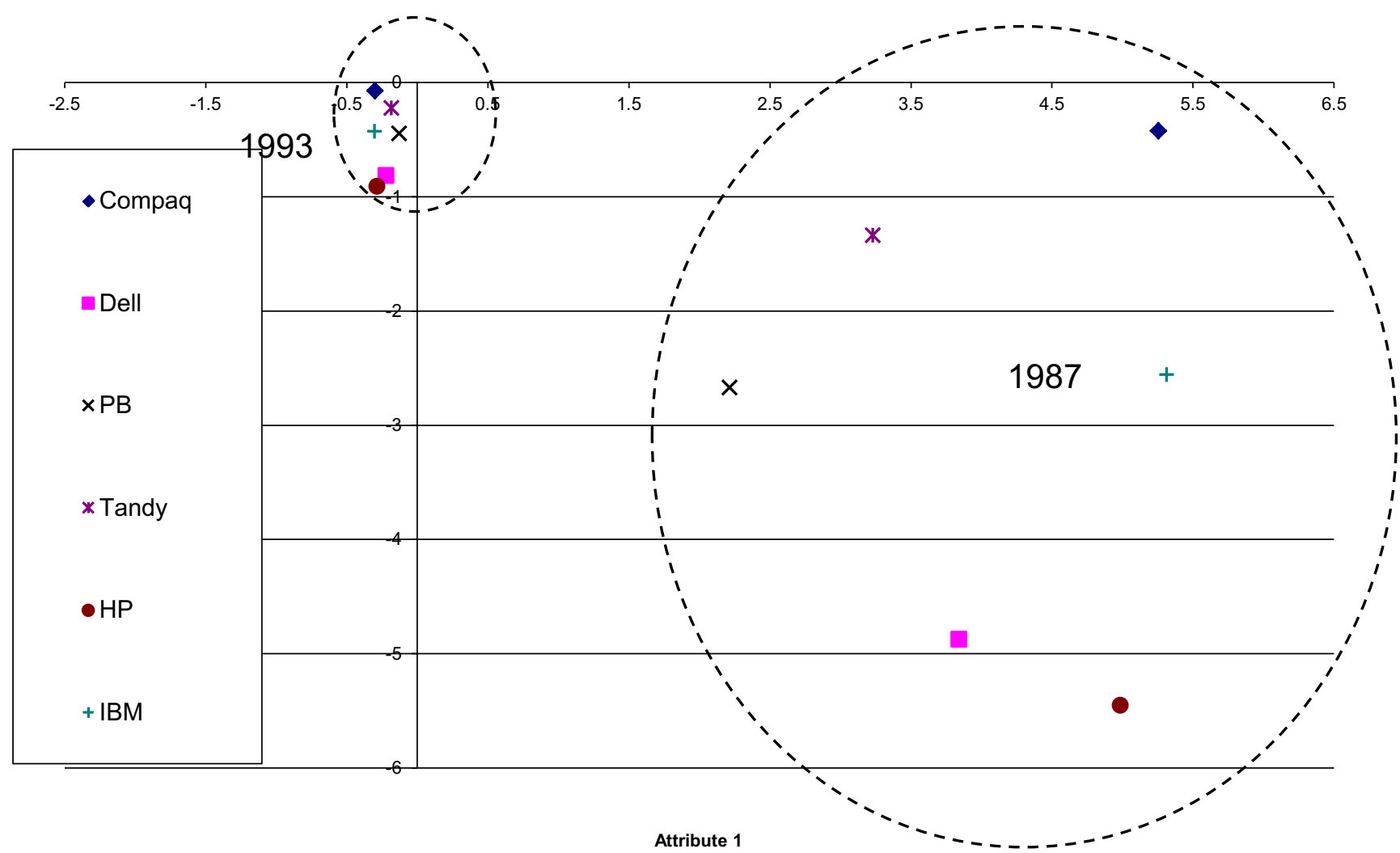

Fig. 7 Estimated changes in total brand preferences, 1987 versus 1993. Attribute 1 ("ease of use/compatibility"), attribute 2 ("service/support/ability to deliver")

counter-factual question: what if the consumer brand preferences were maintained at the level of year 1987? By doing so, we are able to better understand how much of the change in substitutability came from changes in consumer preferences vs. technological innovations or from product line changes within manufacturers. In order to accomplish this, we assume that consumer preferences $c_{t}$ for brand attributes in years from 1983 to 1994 are fixed at their level in 1987, $c_{87}$. Using this, we compute the price elasticities again as described above. The results are reported in columns 5-7 in Table 3.

In general, holding the consumer preferences at their 1987 levels produced own-price elasticities that are much smaller
Table 3 Comparison of ownand cross-price elasticities (timevarying preferences vs. fixed preferences vs. time-invariant preferences)

\begin{tabular}{|c|c|c|c|c|c|c|}
\hline \multirow[b]{2}{*}{ Year } & \multicolumn{3}{|c|}{ Time-varying preferences (proposed model) } & \multicolumn{3}{|c|}{ Preferences fixed at 1987 levels } \\
\hline & Own-Elast & $\begin{array}{l}\text { Cross-Elast } \\
\text { (inside brands) }\end{array}$ & $\begin{array}{l}\text { Cross-Elast } \\
\text { (other brands) }\end{array}$ & Own-Elast & $\begin{array}{l}\text { Cross-Elast } \\
\text { (inside brands) }\end{array}$ & $\begin{array}{l}\text { Cross-Elast } \\
\text { (other brands) }\end{array}$ \\
\hline 1983 & -2.64 & 0.02 & 0.01 & -4.17 & 0.15 & 0.00 \\
\hline 1984 & -4.54 & 0.31 & 0.01 & -6.60 & 0.41 & 0.01 \\
\hline 1985 & -4.49 & 0.35 & 0.01 & -5.80 & 0.38 & 0.01 \\
\hline 1986 & -3.14 & 0.17 & 0.02 & -3.72 & 0.26 & 0.02 \\
\hline 1987 & -4.80 & 0.43 & 0.02 & -4.80 & 0.43 & 0.02 \\
\hline 1988 & -4.94 & 0.35 & 0.01 & -4.79 & 0.34 & 0.02 \\
\hline 1989 & -5.94 & 0.46 & 0.02 & -6.11 & 0.57 & 0.04 \\
\hline 1990 & -5.45 & 0.35 & 0.01 & -6.64 & 0.58 & 0.05 \\
\hline 1991 & -6.69 & 0.39 & 0.01 & -6.60 & 0.61 & 0.06 \\
\hline 1992 & -8.15 & 0.66 & 0.05 & -5.41 & 0.48 & 0.10 \\
\hline 1993 & -6.46 & 0.60 & 0.04 & -4.25 & 0.41 & 0.10 \\
\hline 1994 & -6.66 & 0.53 & 0.05 & -1.66 & 0.09 & 0.12 \\
\hline
\end{tabular}


since 1992 (comparing column 5 to column 2 in Table 3 ). The implication is that including the change in consumer brand preferences increases the estimated own-price elasticities. Cross-price elasticities between these brands are also smaller in that period (comparing column 6 to column 3 in Table 3). All these imply that the change in consumer brand preferences over time increased brand competition, consistent with all of the evidence presented above. We are now beginning to see a clear picture of evolution over time in this market. Specifically, we see now that the central driver behind the "commoditization" of the PC industry was the changes in preferences for brand attributes over the period of the study.

\section{Conclusions and Some Extended Research Questions}

In this research, we document the evolving changes in consumer brand preferences that occurred due to the technological innovations in the supply chain and competition across manufacturers. As a result of this, the market structure or consumer preferences are likely to change significantly over time, hence generating strategic responses from firms such as the intense price competition we observed in the PC industry in the 1990s. We use a factor-analytic modeling approach that builds on previous studies such as Elrod [9] and Elrod and Keane [10]. We show that the model assuming the evolution of consumer preference weights for brand attributes can explain the observed changes in the market share of PC manufacturers and their pricing competition over years. There are several contributions in this paper. First, we develop a methodology to infer the timevarying changes in consumer preferences over time from market-level aggregate data. Second, we study of the impact of the changes in consumer brand preferences on the degree of substitutability between different manufacturer brands over time. Finally, by identifying the above changes, we can gain better insights into the nature of competition in the rapidly evolving technology markets.

Still, there are future research topics that need to be explored. First, we find that there is evidence of increasing substitutability across brands that explain the convergence in prices across manufacturers of personal computers beginning in the early 1990s. Future research should structurally model the implications of the changes in consumer preferences on channel power between, say, downstream manufacturers and upstream suppliers such as Intel and Microsoft as well optimal pricing policy used by these firms as technology develops. Furthermore, we demonstrate that the market structure changed significantly over time and that the dispersion of brand positions decreased dramatically as perceived by consumers. Future research could examine the roles of some key factors, such as advertising vs. R\&D efforts that generate the observed changes in brand competitive positions.

\section{References}

1. Allenby GM, Lenk PJ (1995) Reassessing brand loyalty, price sensitivity, and merchandising effects on consumer brand choice. J Bus Econ Stat 13(3):281-289

2. Bayus B, Putsis W (1999) Product proliferation: an empirical analysis of product line determinants and market outcomes. Mark Sci 18(2):137-153

3. Berry S, Levinsohn J, Pakes A (1995) Automobile prices in market equilibrium. Econometrica 63(4):841-890

4. Bresnahan TF, Greenstein S (1997) "Technological competition and the structure of the computer industry," manuscript.

5. Carroll JD, De Soete G, DeSarbo WS (1990) Two stochastic multidimensional choice models for marketing research. Decis Sci 21: 337-356

6. Chib S, Greenberg E (1995) Hierarchical extensions of sur models with extensions to correlated serial errors and time-varying parameter models. J Econ 68:339-360

7. Chintagunta P (1994) Heterogeneous logit model implications for brand positioning. J Mark Res 31(May):304-311

8. Putsis W, Dhar R (2009) "Category expenditure and promotion: can private labels expand the pie?" unpublished manuscript, London Business School.

9. Elrod T (1998) Choice map: inferring a product-market map from panel data. Mark Sci 7(1):21-40

10. Elrod T, Keane M (1995) A factor-analytic probit model for representing the market structure in panel data. J Mark Res 32(1): $1-16$

11. Erdem T (1996) A dynamic analysis of market structure based on panel data. Mark Sci 15(4):359-378

12. Erdem T, Winer R (1999) Econometric modeling of competition: a multi-category choice-based mapping approach. J Econ 89(1-2): $159-175$

13. Gordon BR (2009) A dynamic model of consumer replacement cycles in the PC processor industry. Mktg Sci 28(5):846-867

14. Lachaab M, Ansari A, Jedidi K, Trabelsi A (2006) Modeling preference evolution in discrete choice models: a Bayesian state-space approach. Quant Mark Econ 4:57-81

15. Langlois R (1992) External economies and economic progress: the case of the microcomputer industry. Bus Hist Rev 66:1-50

16. Putsis W, Bayus B (2001) An empirical analysis of firm product line decisions. J Mark Res 38(1):110-118

17. Seetharaman PB (2004) Modeling multiple sources of state dependence in random utility models of brand choice: a distributed lag approach. Mark Sci 23(2):234-242

18. Smith RJ (1992) Non-nested tests for competing models estimated by generalized method of moments. Econometrica 60(4):973-980

19. Steffens J (1994) New games: strategic competition in the PC revolution. Pergamon Press, New York

20. Van Heerde H, Mela CF, Manchanda P (2004) The dynamic effect of innovation on market structure. J Mark Res 41(2): $166-183$ 